\title{
Fakten versus Fakes: \\ Was Fact-Checking als Maßnahme gegen Desinformation leisten kann und was nicht ${ }^{1}$
}

Alexander Sängerlaub

Abstract

Fact-Checking ist eine notwendige neue journalistische Form in Zeiten, in denen sich ein grundlegendes Paradigma in unseren digitalen Öffentlichkeiten diametral umgekehrt hat: Früher wurde erst verifiziert, dann veröffentlicht. Bei Facebook, Twitter und Co. wird oft erst veröffentlicht, dann vielleicht noch verifiziert. Damit wird die mögliche Wirksamkeit des FactCheckings als Maßnahme gegen Desinformation schon eingeschränkt, denn es gibt zahlreiche strukturelle Probleme - von der Geschwindigkeit der »Fake News" die meist längst über alle Berge sind, bis zur Frage, ob es überhaupt die erreicht, die es brauchen. Vier Herausforderungen des FactChecking werden daher in diesem Beitrag beschrieben: 1) Mangelnde Reichweite, 2) begrenzte Wirkung, 3) ungünstige Plattformlogik sowie 4) die Post-Truth-Era. Den Restriktionen zum Trotz: Als ein Puzzlestück unter vielen ist Fact-Checking in der Bekämpfung von Desinformation unverzichtbar.

Fact-Checking: Wirksames Mittel gegen Desinformationen?

Vor sieben Jahren wurden die sozialen Netzwerke noch als Leuchttürme der vielversprechenden neuen digitalen Öffentlichkeit gepriesen. Sie galten als die Plattformen, die ein altes Paradigma grundlegend aufzulösen schienen: »Pressefreiheit ist die Freiheit von 200 reichen Leuten, ihre Meinung zu verbreiten«, sagte der FAZ-Journalist Paul Sethe vor 30 Jahren.

1 Dieser Text ist eine neu überarbeitete und aktualisierte Version des 2018 erschienenen Papiers der Stiftung Neue Verantwortung: »Feuerwehr ohne Wasser? Möglichkeiten und Grenzen des Fact-Checkings als Mittel gegen Desinformation« (Sängerlaub 2018c). Ein Dank für die Mitarbeit am Text geht an Alina Rathke. 
»Heute ist die Pressefreiheit die Freiheit von rund drei Milliarden Menschen mit Internetzugang ihre Meinung ins Netz zu stellen« (Wolf, 2013), ergänzt der österreichische Journalist Armin Wolf und beschreibt damit den Umbruch, den wir heute als digitalen Strukturwandel der Öffentlichkeit bezeichnen.

Dass heute jede*r am öffentlichen Diskurs teilnehmen kann, ist zunächst ein pluralistischer und urdemokratischer Mehrwert, der das Zeitalter der digitalen Kommunikation auszeichnet. Die andere Seite der Medaille sind allerdings völlig neue Grundvoraussetzungen und Fähigkeiten, die von den Nutzer*innen verlangt werden, um souverän in digitalen Öffentlichkeiten zu navigieren. Darüber hinaus werden aber gerade im öffentlichen Diskurs vor allem die Schattenseiten des, wenn man es so nennen mag, digitalen Strukturwandels der Öffentlichkeit deutlich, wie die zunehmenden Räume für die Verbreitung von Desinformationen zeigen. Vor allem Extremist*innen, Nationalist*innen und Populist*innen machen sich diese neue Dynamik zu eigen und setzen vermehrt auf Manipulation durch die Herausbildung eigener - in deren Selbstverständnis sogenannter alternativer - Informationskanäle, die sich vor allem dadurch auszeichnen, dass klassische Medien und deren Einordnung, Verifizierung und Kontextualisierung strategisch ausgeschaltet werden.

Der signifikanteste Unterschied zur analogen Ära liegt dabei in einer simplen, aber entscheidenden Veränderung: Wurden im analogen Zeitalter Aussagen, bevor sie in die Öffentlichkeit gelangten, auf ihre Faktizität geprüft, verschiebt sich heute in den sozialen Netzwerken ihre Überprüfung ins Nachhinein: Erst wird heute publiziert, dann verifiziert - sofern denn die Faktenchecker überhaupt rechtzeitig Desinformation erkennen und enttarnen (Stichwort: Debunking). Dies gilt dabei weniger für journalistische Qualitätsmedien, die online Artikel veröffentlichen, sondern vor allem für einzelne Akteur*innen wie Privatpersonen oder auch politische Gruppierungen.

Währenddessen hat sich die Geschwindigkeit der Erstellung und Verbreitung von Nachrichten massiv erhöht und folgt, vor allem innerhalb der Plattformen, teils neuen Regeln der Aufmerksamkeit. Dabei werden die affektgeladenen Plattformlogiken von Facebook und Co. ausgenutzt, um nicht nur bewusst Falschinformationen (= Desinformation) zu platzieren, sondern auch medial ungefiltertes Agenda Setting und Framing zu betreiben. Was die deutsche Bundestagswahl 2017 betrifft, vor allem durch Rechtspopulisten (siehe Sängerlaub zur Bundestagswahl 2017 in dieser Publikation). Diskurse werden so maßgeblich beeinflusst, auch um das Vertrauen in Medien und Demokratien zu schwächen und die eigenen 
Wähler*innen zu mobilisieren. Dass das Medienvertrauen insbesondere in digitalen Öffentlichkeiten, die von politischen Unruhen betroffen sind, schwindet, bestätigen auch Zahlen aus dem Digital News Report 2019. In Europa betraf dies insbesondere Frankreich, wo nur noch 24 Prozent der Befragten angaben, dass sie den »meisten Medien meistens" vertrauten. Das sind 11 Prozent weniger als noch im Vorjahr (Reuters Institute, 2019). Diese Entwicklung wird vor allem auf die Berichterstattung um die Gelbwesten-Proteste zurückgeführt.

Eine Antwort auf diese veränderte Situation ist das sogenannte Fact-Checking, das seine Blüte nach den letzten US-Wahlen auch in Europa erlebt hat, um der scheinbar zunehmenden Dichte an »Fake News«, vor allem in Wahlkämpfen, etwas entgegenzusetzen. Liest man die Handlungsempfehlungen der EU gegen Desinformation (Europäische Kommission, 2018), hat das Fact-Checking den Ruf der Feuerwehr erlangt, um »Fake News«Brände in den sozialen Netzwerken und außerhalb zu löschen. Die EUKommission hebt in ihrem im April 2018 veröffentlichten Papier »Tracking online disinformation: a European Approach" das Fact-Checking als »integral element in the media value chain « (Europäische Kommission, 2018) hervor. Bereits vor der Wahl Trumps im Jahr 2016 wird in einem Report des Reuters Institute vom Fact-Checking als einer notwendig gewordenen »democratic institution « (Graves \& Cherubini 2016,6) gesprochen.

Doch der Erfolg des nachträglichen Fact-Checkings hat sich bisher noch nicht empirisch bestätigt: So verbreiten sich beispielsweise Unwahrheiten im Netz gleich sechsmal schneller als wahre Nachrichten, konstatiert die Studie von Vosoughi, Roy \& Aral (2018) vom MIT (Massachusetts Institute of Technology), bei der über 125.000 auf Twitter geteilte Beiträge untersucht wurden (zwei Drittel von ihnen waren dabei faktisch falsch).

Auch in den von uns untersuchten zehn Case-Studies zur deutschen Bundestagswahl (Sängerlaub et al., 2018a) wiederholte sich fast ausnahmslos das gleiche Muster: »Fake News« erzielen wesentlich höhere Reichweiten als deren Richtigstellungen, vor allem wenn an deren Verbreitung auch noch große Medienhäuser beteiligt sind. Wird dann doch erfolgreich Debunking betrieben (werden »Fake News« also als solche entlarvt), kommt es allerdings anscheinend nicht bei den Nutzer*innen der Plattformen an und - selbst wenn - wird es dort wohl ignoriert (Sängerlaub et al., 2018a).

Es stellt sich daher die Frage, ob nachträgliches Fact-Checking überhaupt das geeignete Mittel gegen Desinformationen ist? Kommt es dort an, wo die Desinformation rezipiert wurde? Und wenn dem so ist, kann es wiederum die Einstellung derjenigen, die vorher die sogenannten Alternativen Fakten konsumiert haben, nachhaltig beeinflussen? 
In diesem Beitrag werden sowohl Potenzial als auch Limitationen des FactCheckings diskutiert. Welche systemischen und institutionellen Grundvoraussetzungen braucht es für ein erfolgreiches Fact-Checking?

\section{Herausforderungen für Fact-Checking}

Mehr als die Hälfte der Befragten aus den 38 im Digital News Report 2019 untersuchten Ländern gab an, besorgt zu sein, nicht auseinanderhalten zu können, welche Nachrichten im Internet wahr und welche falsch seien (Reuters Institute, 2019). Insbesondere im Vereinigten Königreich (70 Prozent, 2018 noch 58 Prozent) sowie in Frankreich (67 Prozent, 2018 noch 62 Prozent) haben die Werte im Vergleich zur Vorjahreserhebung stark zugenommen. In beiden Ländern waren die digitalen Öffentlichkeiten mit politischen Unruhen (Brexit, Gelbwesten-Proteste) und der in diesem Zusammenhang verbreiteten Desinformationskampagnen konfrontiert.

International 114 aktive Fact-Checking-Organisationen zählte 2017 eine vom Duke Reporters' Lab durchgeführte Studie (Stencel, 2017). Auch Google und Facebook haben auf die Diskussion um Desinformationen reagiert: Google führte im Jahr 2016 ein sogenanntes Faktencheck-MarkupTool (Google, 2016) ein, ein Add-On mit dem Artikel auf Google News direkt auf ihre Richtigkeit überprüft werden können. Auch Facebook kooperiert länderspezifisch mit unterschiedlichen Fact-Checking-Organisationen. In Deutschland sind das der $d p a$-Faktencheck sowie das RechercheNetzwerk Correctiv (Facebook, o. A. a). Einige Implikationen, wie gutes Fact-Checking funktioniert, geben Institutionen wie das International FactChecking Network Poynter, die einen Code of Principles für die Arbeit von Fact-Checking-Organisationen entwickelt haben (Poynter, 2019). Zu den Merkmalen gehören unter anderem Transparenz in Bezug auf Quellen, Methode, Finanzierung sowie Fairness und Unvoreingenommenheit.

Eine simple, aber wesentliche Limitation des nachträglichen Fact-Checkings liegt in der Logik der Sache: Es kann weder die Produktion noch die Rezeption von Desinformationen im Wesentlichen verhindern. Vier Kriterien, die die Wirkung des Fact-Checkings - selbst eines hochwertig durchgeführten - einschränken und erschweren, sollen im Folgenden genauer ausgeführt werden.

Mangelnde Reichweite: Fact-Checking hat bei der Frage möglicher Reichweite mit zwei Hürden zu kämpfen: 1) der Zeitverzögerung in der Veröffentlichung zwischen Desinformation und Debunking sowie 2) der 
Eigendynamik der Nachrichtenlogik, die »Fake News« fast immer reichweitenstärker macht.

Begrenzte Wirkung: Eine Vielzahl von Studien (unter anderem Nyhan \& Reifler, 2010; Lewandowsky; Ecker; Seifert; Schwarz \& Look, 2012) belegt, dass selbst sehr gewissenhaftes Fact-Checking kaum Wirkung erzielt. Gerade im politischen Bereich wird versucht, gegen bestehende Weltanschauungen anzukämpfen - der Einstellungswandel ist jedoch psychologisch gesehen ein komplexer und ressourcenintensiver Vorgang. Dazu kommt, dass Fact-Checking in bestimmten Konstellationen sogar kontraproduktiv sein kann und damit bestehende Ansichten verfestigt werden (Backfire-Effekt), auch wenn es hier unterschiedliche Meinungen gibt (Wood \& Porter, 2018).

Ungünstige Plattformlogik: Auch die Blaupause der Plattformen sowie die damit entstehende Aufmerksamkeitsökonomie wirken eher ungünstig auf die Güte der Information: Affektive Reaktionen (wie Wut, Freude und Trauer) als Grundlogik der Nutzungsinteraktion in den Plattformen sowie das einheitliche Design der den Nutzer*innen dargebotenen Informationen unterschiedlicher Qualität begünstigen Desinformationen (Bestätigungsfehler) zusätzlich, aber auch Formen entgrenzter Kommunikation (wie etwa Hate Speech).

Post-Truth-Era: Mittlerweile wird diskutiert, welche sozialen, ökonomischen und politischen Verwerfungen darüber hinaus Desinformationen begünstigen. Diese unter dem Begriff Post-Truth-Era oder dem sogenannten postfaktischen Zeitalter zusammengefassten Entwicklungen sind mehr als nur Schlagworte, sondern auch empirisch nachweisbare Veränderungen in unseren liberal-demokratischen Gesellschaften.

\section{Faktor Reichweite: Zeit und Nachrichtenlogik erschweren Erfolg}

Die größte Hürde für den Erfolg des Fact-Checkings liegt im Zeitversatz zwischen der Veröffentlichung einer Falschinformation und deren Richtigstellung. Je schneller man reagiert, desto höher ist die Wahrscheinlichkeit, dass sich falsche Informationen nicht weiterverbreiten. Doch die Praxis zeigt: Zwischen der Veröffentlichung einer »Fake News« und deren Korrektur können bisweilen schon einmal 24 bis 72 Stunden liegen. Sowohl, weil das Richtigstellen teils aufwendige journalistische Recherche erfordert, als auch, weil die Verbreitung der Falschinformation erst viel später festgestellt wird (Sängerlaub et al., 2018a). 
Die Fact-Checking-Institutionen können somit erst im Nachhinein überprüfen und reagieren. Je nachdem, wie aufwendig die Gegenrecherche ist, braucht es Zeit, um die sogenannten Alternativen Fakten als solche zu enttarnen. In der Zwischenzeit haben sich »Fake News«, gerade wenn sie negative oder überraschende Informationen beinhalten, bereits viral verbreitet. Die oben erwähnte Studie des MIT ergänzt hierzu:

»Wir haben die Meldungen in Übereinstimmung mit sechs unabhängigen Organisationen als wahr oder falsch klassifiziert. Unwahrheiten verbreiteten sich in allen Informations-Kategorien schneller, weiter und tiefgehender als die Wahrheit. Die Effekte waren ausgeprägter für politische Falschmeldungen als für Falschmeldungen über Terrorismus, Naturkatastrophen, Wissenschaft, städtische Legenden oder Finanzen. Wir haben ermittelt, dass falsche Meldungen überraschender sind als wahre. Das impliziert, dass Menschen mehr dazu neigen, überraschende Informationen zu teilen«, übersetzt Netpolitik.org. (Laaß, 2018)

Für die Kommunikationswissenschaft ist das wahrlich keine Überraschung, ist diese doch ein Indikator für die Relevanz einer Nachricht und ihrem sogenannten Nachrichtenwert. Ein weiterer Faktor ist beispielsweise die Emotionalität einer Meldung, je eher Emotionen aktiviert werden, desto höher ist ebenfalls ihre Verbreitungswahrscheinlichkeit (Wineburg, McGrew, Breakstone \& Ortega, 2016).

Für Fact-Checking-Institutionen ist es umso herausfordernder, mit einer Nachricht, dass etwas nicht richtig ist, den gleichen Nachrichtenwert zu erzielen; heißt, diese Richtigstellung journalistisch so schmackhaft zu machen, dass sie auch in den sozialen Netzwerken eine hohe Verbreitung erfährt. Sind Geschwindigkeit und Nachrichtenwert hoch - bei gleichzeitiger Akkuratheit - begünstigt das die Reichweite des Debunking und damit auch seinen Erfolg.

Eine im Bundestagswahlkampf 2017 verbreitete Falschinformation auf Grundlage einer gefälschten Dienstanweisung des nordrhein-westfälischen Innenministeriums, dass Straftaten von Migranten von der Polizei vertuscht werden sollen (Der Westen, 2017), konnte auch deshalb schnell enttarnt werden, weil das zuständige Innenministerium auf Twitter das Dokument sehr schnell als falsch deklarierte. Ein professioneller Umgang von öffentlichen Behörden und Institutionen ist somit in der digitalen Öffentlichkeit ein Schlüssel bei der Abwehr von Desinformationen.

Auch das sehr erfolgreiche Debunking in der gesamten deutschen digitalen Öffentlichkeit in Bezug auf den Tweet Donald Trumps, der im Juni 2018 behauptete, dass die Kriminalität in Deutschland aufgrund der Migration um zehn Prozent gestiegen sei, hat sicherlich viel mit der Konstellation der Akteure - und der damit verbundenen Erwartungshaltung der deutschen 
Öffentlichkeit an die Faktizität der Tweets des US-Präsidenten zu tun (Sängerlaub et al., 2018b).

Neben der Zeit und Nachrichtenlogik spielt auch die Ausspielung des Faktenchecks, also die Frage, auf welcher Seite oder Plattform er stattfindet, eine wichtige Rolle, wenn es darum geht, Reichweite zu erzielen. Seiten wie der Faktenfinder des ARD-Nachrichtenangebots tagesschau.de erreichen über ihre Webseite zwar viele Nutzer*innen, finden damit aber nicht dort statt, wo Falschinformationen in der Regel geteilt werden. Dort, wo besonders viele »Fake News« zirkulieren und geglaubt werden, ist zudem auf Nutzer*innenseite die Glaubwürdigkeit in die klassischen Medien eher gering (Johannes Gutenberg-Universität Mainz, 2018).

Facebook geht daher einen anderen Weg und arbeitet mit dem deutschen Recherche-Netzwerk Correctiv zusammen, das über seine offizielle Facebook-Seite rund 30.000 Nutzer*innen erreicht. Seit 2019 ist auch die dpa mit Fact-Checkern an Bord. Die Arbeit liegt hauptsächlich darin, innerhalb der Plattform durch Nutzer*innen gemeldete oder durch regelmäßiges $M o$ nitoring entdeckte »Fake News « zu markieren, zu debunken oder auf andere (korrigierende) Berichterstattung aus vertrauenswürdigen Quellen hinzuweisen. Darüber hinaus arbeitet Facebook kontinuierlich am Algorithmus des Newsfeeds. So wurde Anfang 2018 die Menge an Nachrichten in den Newsfeeds weltweit reduziert. Derzeit wird auch an der Einschätzung der Quellen durch die Schwarmintelligenz der Nutzer*innen gearbeitet, um herauszufinden, welche Medienmarken vertrauenswürdig sind und um diese Informationen bei der Ausspielung von Nachrichten zu berücksichtigen. Wird ein Beitrag von einem Fact-Checker als falsch markiert, reagiert zudem der Algorithmus darauf und vermindert die Reichweite eines Beitrages (Correctiv, 2018).

Ebenso wie »Fake News « besonders große Reichweiten aufbauen, wenn etablierte Medien sie teilen, verhält es sich auch beim Debunking. So kommen die reichweitenbedingt erfolgreichen Faktenchecks oft von den Nachrichtenangeboten im Netz, die ohnehin hohe Reichweiten erzielen (wie zum Beispiel sueddeutsche.de).

\section{Faktor Wirkung: Alternative Fakten halten sich hartnäckig}

Wie wirksam Fact-Checking tatsächlich ist, hängt von verschiedenen Faktoren ab. Ein wichtiges Merkmal ist unter anderem eine gelungene Ansprache und Tonalität. So zeigt beispielsweise die Neurolinguistin Elisabeth Wehling, dass es wichtig sei, es nicht bei der Falschinformation zu belassen, 
sondern neue Narrative beim Debunking zu finden (Wehling, 2016). Dies erhöhe nachweislich den Effekt des Debunking (Chan, Jones, Hall Jamieson \& Albarracìn, 2017). Auch eine grafische Unterstützung sei darüber hinaus hilfreich (Nyhan \& Reifler, 2010). Des Weiteren sei es wichtig zu erklären, wie der Fehler zustande kam, beziehungsweise, wer welches Narrativ - und mit welcher Absicht - etabliert hat. Außerdem darf laut Wehling (2016), das Fact-Checking das Weltbild des Gegenübers nicht zu radikal herausfordern, um den sogenannten Backfire-Effekt zu verhindern - gerade im Zusammenhang mit politischen Desinformationen ist dies aber immer wieder der Fall.

Wichtig ist hierbei, dass nicht jedes Publikum identisch reagiert. Verschiedene Strategien können bei verschiedenen Publika zu unterschiedlichen Wirkungen führen: Es gibt den Teil des Publikums, der Fakten gegenüber offensteht, Vertrauen in Medienangebote hat und das Fact-Checking aufnimmt, aber es vermutlich aufgrund seiner ohnehin vielfältigen Mediennutzung nur bedingt benötigt. Und am anderen Ende der Publikumsskala sind Menschen mit massiven Vorbehalten gegenüber den Medien, die für Fact-Checking, gerade durch diese Quellen, ohnehin nicht offen sind und sich eher sogenannten alternativen Medienangeboten zuwenden. Bei ihnen kann Fact-Checking zu diesen eben erwähnten negativen Backfire-Effekten führen, da die Torpedierung des eigenen Weltbildes zu einer Art »Jetzt-erstRecht«-Mentalität führt (Peter \& Koch, 2015).

Der sogenannte Backfire-Effekt wurde erstmals 2010 durch Brendan Nyhan und Jason Reifler beschrieben und konnte auch in anderen Studien nachgewiesen werden (Lewandowsky et al., 2012). Erwähnenswert ist in diesem Zusammenhang ebenso die Studie von Oscar Barrera Rodriguez und Kolleg*innen (Barrera Rodriguez et al., 2017), die den Erfolg des FactCheckings während der französischen Präsidentschaftswahl unter Anhänger*innen von Marine Le Pen untersucht haben. Diese nutzte regelmäßig sogenannte Alternative Fakten, um ihre Anhänger*innen zu mobilisieren. Sie konnten nicht nur zeigen, dass das hochgradig persuasive Mobilisieren mit diesen sogenannten Alternativen Fakten funktionierte, sondern auch, dass das Fact-Checking im Grunde so gut wie keine Wirkung entfaltete: »Fact checking does nothing to undo these effects despite improving factual knowledge of voters. Being exposed only to official facts also backfires on voting intentions, as it increases political support for Marine Le Pen« (Barrera Rodriguez et al., 2017).

Fabiana Zollo und Kolleg*innen kommen zu dem Schluss, dass algorithmisch basierte Lösungen sowie Debunking-Kampagnen wenig hilfreich seien, zumal die Persistenz von Weltanschauungen sogar eher dazu führt, 
dass das Debunking als Bedrohung wahrgenommen und somit das Vertrauen in die eigene Echokammer eher bestärkt werde (Zollo et al., 2017). Der Weg in die Echokammer ist dabei auch empirisch nachzeichenbar: Die unheilige Allianz aus geringem Medien- und Institutionenvertrauen, geringer Medienkompetenz und die starke Nutzung von Social Media als alternativer, primärer Informationsquelle, steigern stark die Wahrscheinlichkeit, auf »Fake News« hereinzufallen sowie für Debunking nicht zugänglich zu sein (De keersmaecker \& Roets, 2017; Sängerlaub, 2017).

Trotz alledem sind die vielzitierten Echokammern allen empirischen Erkenntnissen nach nicht der Normalfall. Sie entsprächen demnach nicht der alltäglich erlebten Medienrealität der Vielzahl der Internetnutzer*innen. Studien zeigen sogar im Gegenteil, dass die meisten Nutzer*innen mit einer ganzen Bandbreite politischer Standpunkte konfrontiert würden (Stark, Magin \& Jürgens, 2017; Bakshy, Messing \& Adamic, 2015). Dies sei durch die vielen Diskussionen zu Filterblasen und Echokammern, die zur Absolution neigen, zuweilen überdeckt (Garrett, 2017).

\section{Faktor Social-Media-Plattformen: Kognitionsprobleme werden noch unterstützt}

Die Art und Weise, wie Menschen Nachrichten und Informationen via beispielsweise Facebook konsumieren, scheint dabei zusätzliche Kognitionsprobleme zu verursachen. In der Liste der Wahrnehmungsfehler ist dabei vor allem der Confirmation Bias (Bestätigungsfehler) hervorzuheben:

Wissenschaftler haben beobachtet, dass viele Leute eher zum Zeitvertreib in sozialen Netzwerken surfen, aber weniger, um gezielt Nachrichten zu lesen. Sie sind entspannter, nehmen Inhalte eher en passant wahr, lesen oft nur Schlagzeilen. Auf den Link zum ausführlichen Artikel klicken sie nicht so oft. In solchen Phasen neigen Menschen dazu, eher Informationen wahrzunehmen, die ihr eigenes Weltbild bestätigen. Die Gefahr: Passt eine ,Fake News` zum Weltbild eines Nutzers, wird sie weniger oft hinterfragt und eher geglaubt. (Faktenfinder, 2017)

Dazukommt, dass sich per Design in der Timeline der Nutzer*innen verschieden dargebotene Informationshäppchen vermischen, die sich optisch kaum voneinander unterscheiden: Werbeanzeigen, Gerüchte, private Statusmeldungen, Nachrichten, Memes und Desinformation werden im gleichen Design dargeboten - in der Flüchtigkeit der Konsumption ist selbst bei hoher Medienkompetenz das Unterfangen schwierig, die Informationsgüte dauernd mitzubeurteilen. Weitere Plattformeffekte ergeben sich durch die Fokussierung auf Emotionen - Like, Lachen, Love, Trauer, Wow und Wut - mit denen bei Facebook auf Artikel reagiert werden kann. Damit forciert 
die Plattform mehr affektive Reaktionen als kognitive Auseinandersetzungen mit Kontext und Inhalten. (Journalistische) Inhalte passen sich an diese Logik wiederum an, beispielsweise durch Clickbaits, also emotionalisierte und überspitzte Überschriften und Zusammenfassungen, die wiederum die Interaktion der Nutzer*innen forcieren sollen. Insgesamt kann man von einer eher ungünstigen Aufmerksamkeitsökonomie sprechen, die, so die Vermutung, wiederum hohe Informations- und Nachrichtenkompetenzen der Nutzer*innen erfordert, um souverän mit diesen Widrigkeiten umgehen zu können.

Dieser Informationsraum scheint daher gleichermaßen auch ein Desinformationsraum zu sein, in dem ebenso verschwörungstheoretische Inhalte die passende Community, beispielsweise in geschlossenen Gruppen, finden:

Conspiracy related contents become popular because they tend to reduce the complexity of reality and convey general paranoia on specific objects and are more affordable by users. On our perspective the diffusion of bogus content is someway related to the increasing mistrust of people with respect to institutions, to the increasing level of functional illiteracy - i.e., the inability to understand information correctly - affecting western countries as well as the combined effect of confirmation bias at work on a enormous basin of information where the quality is poor. (Zollo et al., 2015, 11)

Welche Konsequenz ergibt sich daraus? Zugespitzt könnte man meinen, dass vor allem Facebook schlicht zur Informationsaufnahme im Sinne einer Rezeption von qualitativ hochwertigen Nachrichten ungeeignet scheint. Die anhaltende Diskussion in der Öffentlichkeit über die Qualität der Informationen, die einen über soziale Netzwerke erreichen, schlägt sich nun genauso in den Nutzungszahlen nieder wie die Anpassung des Algorithmus. Die Zahlen derjenigen, die Facebook beispielsweise als Informationsquelle für Nachrichten nutzen, ist seit 2017 erstmals rückläufig, ermittelte der Digital News Report 2018 des Reuters Institute. Die Plattformen reagieren wiederum ihrerseits, indem versucht wird, die Vertrauenswürdigkeit gegenüber einer Quelle als Indikator für die Ausspielung von Inhalten stärker zu berücksichtigen:

We find that the move to distributed content via social media and aggregators has been halted - or is even starting to reverse, while subscriptions are increasing in a number of countries. Meanwhile notions of trust and quality are being incorporated into the algorithms of some tech platforms as they respond to political and consumer demands to fix the reliability of information in their systems. (Reuters Institute, 2018, 10) 
Stephan Lewandowsky, Kognitionspsychologe an der Universität Bristol in Großbritannien, und seine Kollegen, gehen sogar so weit zu sagen, dass dem Fact-Checking die langfristigen Kommunikationsmittel fehlen, um als Maßnahme gegen Desinformationen geeignet zu sein. Im Kontext der PostTruth-Era sieht er das Fact-Checking mehr als Bekämpfung eines Symptoms anstatt der Ursache (Lewandowksy, Ecker \& Cook, 2017). Im Rahmen eines größeren Theoriegerüsts, das den politischen, technologischen und sozialen Kontext berücksichtigt, zeigen sie auf, welche Ursachen die PostTruth-Era (postfaktisches Zeitalter) hat.

Aus der normativen Ableitung der Notwendigkeit einer gut-informierten Öffentlichkeit in liberalen Demokratien definieren sie eine Vielzahl von Faktoren, die ein postfaktisches Zeitalter begünstigen: der hohe ökonomische Druck in den Redaktionen, der wachsende Rückgang des sozialen Kapitals, die zunehmenden ökonomischen Ungleichheiten, die daraus resultierende Polarisation von Gesellschaften oder der Rückgang des Vertrauens in die Wissenschaft (Lewandowsky et al., 2017) sind einige Punkte, die hier exemplarisch angeführt werden.

Damit wird deutlich, dass Fact-Checking als isolierte Maßnahme keine alleinige Antwort gegen Desinformationen sein kann. Es muss stattdessen immer in einem breiteren gesellschaftlichen Kontext betrachtet werden von der Stabilität des politischen Systems und dessen Umgang mit der Desinformation bis hin zur Vielfalt und Qualität innerhalb des Medienangebots. Eine wichtige Rolle spielt zudem die (digitale) Medienkompetenz der Bürger*innen, die es zu stärken gilt. Plattformen und soziale Netzwerke, die Räume für Desinformationen öffnen, müssen stärker in die Pflicht genommen werden, wie bei Facebook bereits geschehen: Dort hat der öffentliche und politische Druck dafür gesorgt, dass nun sichtbar ist, welche Seiten, welche Werbung schalten. Zudem solle politische Werbung im besonderen Maße hervorgehoben werden (Facebook o. A. b).

\section{Fazit: Fact-Checking ist notwendig, aber hat Limitationen}

Fact-Checking erfüllt nicht die Erwartungen, die an es gestellt werden. Obwohl es eine wichtige Maßnahme im Kampf gegen Desinformationskampagnen darstellt, kann es nicht das Allheilmittel sein. Anstatt diese Methode als isolierte Strategie gegen die Verbreitung von Desinformationen zu verstehen, muss es im Kontext größerer gesellschaftlicher medialer Umbrüche 
verstanden werden. Trotz der Limitationen, die in diesem Beitrag ausführlich beschrieben wurden, ist es selbstverständlich ebenfalls keine Lösung, wenn Fact-Checking-Organisationen ihre Arbeit einstellen. Stattdessen gilt es das Fact-Checking als hilfreiches Mittel weiter auszubauen und handlungsfähiger zu machen. So ergibt es durchaus Sinn, bestehende Ressourcen zu bündeln und die Fact-Checking-Abteilungen einiger größerer Medienhäuser zusammenzulegen beziehungsweise eine supra-institutionelle nationale Fact-Checking-Organisation zu etablieren.

Damit es aber nicht nur bei einer Symptombehandlung bleibt, ist es unabdingbar die Resilienz und damit vor allem die Informations- und Nachrichtenkompetenz, als spezifische Formen der Medienkompetenz, der Bürger*innen nachhaltig zu stärken.

Viele Falschinformationen sind zudem auf schlecht gemachte Öffentlichkeitsarbeit öffentlicher Einrichtungen - seien es Ministerien, Behörden oder die Polizei - zurückzuführen. Hier muss der Umgang mit den sogenannten neuen Medien besser geschult werden, damit es gar nicht erst zu Missverständnissen kommt, die sich dann als »Fake News« manifestieren können.

\section{Literatur}

Bakshy, Eytan; Messing, Solomon \& Adamic, Lada A. (2015). Exposure to ideologically diverse news and opinion on Facebook. Science, 348(6239), 1130-1132. DOI: 10.1126/science.aaa1160.

Barrera Rodriguez, Oscar; Guriev, Sergei M.; Henry, Emeric. \& Zhuravskaya, Ekaterina (2017). Facts, Alternative Facts, and Fact Checking in Times of Post-Truth Politics. SSRN Electronic Journal. DOI: 10.2139/ssrn.3004631.

Chan, Man-pui Sally; Jones, Christopher R.; Hall Jamieson, Kathleen \& Albarracín, Dolores (2017). Debunking. A Meta-Analysis of the Psychological Efficacy of Messages Countering Misinformation. Psychological Science, 28(11), 1531-1546. DOI: $10.1177 / 0956797617714579$.

Correctiv (2018). Über die Kooperation zwischen CORRECTIV. Faktencheck und Facebook. Abgerufen von https://correctiv.org/faktencheck/ueber-uns/2018/12/17/ ueber-die-kooperation-zwischen-correctiv-faktencheck-und-facebook/. Abgerufen am 20. August 2019.

De keersmaecker, Jonas \& Roets, Arne (2017). »Fake News«. Incorrect, but Hard to Correct. The Role of Cognitive Ability on the Impact of False Information on Social Impressions. Intelligence, 65, 107-110. DOI: 10.1016/j.intell.2017.10.005.

Der Westen (2017). NRW-Polizei kämpft gegen gefälschten Erlass zu Ausländerkriminalität - Von Erika Steinbach verbreitet. Abgerufen von https://www.derwesten.de/ politik/nrw-polizei-kaempft-gegen-gefaelschten-fluechtlingserlassid211599269.html. Abgerufen am 06. August 2019. 
Europäische Kommission (2018). A Multi-Dimensional Approach to Disinformation. Report of the Independent High Level Group on Fake News and Online Disinformation. Abgerufen von https://ec.europa.eu/digital-single-market/en/news/finalreport-high-level-expert-group-fa-ke-news-and-online-disinformation. Abgerufen am 06. August 2019.

Facebook (o. A. a). Faktenprüfung auf Facebook. Das sollten Herausgeber wissen. Abgerufen von https://de-de.facebook.com/help/publisher/182222309230722. Abgerufen am 02. März 2020.

Facebook (o. A. b). Making Ads and Pages More Transparent. Facebook Newsroom. Abgerufen von https://newsroom.fb.com/news/2018/04/transparent-ads-and-pages/. Abgerufen am 06. August 2019.

Faktenfinder (2017). Hohe Reichweite, begrenzte Wirkung. Abgerufen von http:// faktenfinder.tagesschau.de/hintergrund/studien-fake-news-101.html67. Abgerufen am 21. September 2018.

Garrett, R. Kelly (2017). The »Echo Chamber« Distraction. Disinformation Campaigns are the Problem, Not Audience Fragmentation. Journal of Applied Research in Memory and Cognition, 6(4), 370-376. DOI: 10.1016/j.jarmac.2017.09.011.

Google (2016). Labelling Fact-Check Articles in Google News. Abgerufen von https://blog.google/outreach-initiatives/google-news-initiative/labeling-fact-checkarticles-google-news/. Abgerufen am 06. August 2019.

Graves, Lucas \& Cherubini, Federica (2016). The rise of fact-checking sites in Europe. Abgerufen von https://reutersinstitute.politics.ox.ac.uk/sites/default/files/research/ files/The $\% 2520$ Rise $\% 2520$ of $\% 2520$ Fact-Checking $\% 2520$ Sites $\% 2520 \mathrm{in} \% 2520 \mathrm{Eu}-$ rope.pdf.

Johannes Gutenberg-Universität Mainz (2018). Forschungsergebnisse der Welle 2018. Langzeitstudie Medienvertrauen. Abgerufen von https://medienvertrauen.unimainz.de/forschungsergebnisse-der-welle-2018/. Abgerufen am 20. August 2019.

Laaß, Sophie (2018). Studie über Twitter. Menschen neigen zum Verbreiten von Fake News. Abgerufen von https://netzpolitik.org/2018/studie-ueber-twitter-menschenneigen-zum-verbreiten-von-fake-news/. Abgerufen am 06. August 2019.

Leonhardt, David (2017). President Trump's Lies, the Definitive List. The New York Times, 14. Dezember 2017. Abgerufen von https://www.nytimes.com/interactive/ 2017/06/23/opinion/trumps-lies.html. Abgerufen am 08. August 2019.

Lewandowsky, Stephan; Ecker, Ullrich K. H.; Seifert, Colleen M.; Schwarz, Norbert \& Cook, John (2012). Misinformation and Its Correction. Psychological Science in the Public Interest, 13(3), 106-131. DOI: 10.1177/1529100612451018.

Lewandowsky, Stephan; Ecker, Ullrich \& Cook, John (2017). Beyond Misinformation. Understanding and Coping with the »Post-Truth « Era. Journal of Applied Research in Memory and Cognition, 6(4), 353-369. DOI: 10.1016/j.jarmac.2017.07.008.

Nyhan, Brendan \& Reifler, Jason (2010). When Corrections Fail. The Persistence of Political Misperceptions. Political Behavior, 32(2), 303-330. DOI: 10.1007/s11109010-9112-2. 
Peter, Christina \& Koch, Thomas (2015). When Debunking Scientific Myths Fails (and When It Does Not). Science Communication, 38(1), 3-25. DOI: 10.1177/ 1075547015613523.

Poynter (o. A.). IFCN Code of Principles. Abgerufen von https://ifcncodeof principles.poynter.org/know-more/the-commitments-of-the-code-of-principles. Abgerufen am 06. August 2019.

Reuters Institute (2018). Digital News Report 2018. Abgerufen von https://reuters institute.politics.ox.ac.uk/sites/default/files/digital-news-report-2018.pdf.

Reuters Institute (2019). Digital News Report 2019. Abgerufen von https://reuters institute.politics.ox.ac.uk/sites/default/files/inline-files/DNR_2019_FINAL.pdf. Abgerufen am am 26. Februar 2020.

Stark, Birgit; Magin, Melanie \& Jürgens, Pascal (2017). Ganz meine Meinung? Informationsintermediäre und Meinungsbildung - eine Mehrmethodenstudie am Beispiel von Facebook. Eine Expertise der Landesanstalt für Medien Nordrhein-Westfalen (LfM) in Kooperation mit dem Forschungsschwerpunkt Medienkonvergenz der Johannes Gutenberg-Universität Mainz. Düsseldorf: Landesanstalt für Medien Nordrhein-Westfalen (LfM).

Sängerlaub, Alexander (2017). Verzerrte Realitäten. Die Wahrnehmung von »Fake News« im Schatten der USA und der Bundestagswahl. Abgerufen von https://www.stiftung-nv.de/sites/default/files/fake_news_im_schatten_der_usa_ und_der_bundestagswahl.pdf.

Sängerlaub, Alexander (2018). Feuerwehr ohne Wasser? Möglichkeiten und Grenzen des Fact-Checkings als Mittel gegen Desinformation. Abgerufen von https://www.stiftung-nv.de/de/publikation/feuerwehr-ohne-wasser-moeglichkeitenund-grenzen-des-fact-checkings-als-mittel-gegen. Abgerufen am 20. August 2019.

Sängerlaub, Alexander; Meier, Miriam \& Rühl, Wolf-Dieter (2018). Fakten statt Fakes. Verursacher, Verbreitungswege und Wirkungen von Fake News im Bundestagswahlkampf 2017. Abgerufen von https:/www.stiftung-nv.de/sites/default/files/snv fakten_statt_fakes.pdf.

Sängerlaub, Alexander \& Rühl, Wolf-Dieter (2018). Kurzanalyse zu Trumps CrimeTweet in Deutschland. Viel Aufmerksamkeit, wenig Unterstützung. Abgerufen von https://www.stiftung-nv.de/de/publikation/kurzanalyse-zu-trumps-crime-tweetdeutschland-viel-aufmerksamkeit-wenig-unterstuetzung. Abgerufen am 02. März 2020.

Stencel, Mark (2017). International Fact-Checking Gains Ground, Duke Census Finds Duke Reporters' Lab. Abgerufen von https://reporterslab.org/international-factchecking-gains-ground/. Abgerufen am 06. August 2019.

Vosoughi, Soroush; Roy, Deb \& Aral, Sinan (2018). The spread of true and false news online. Science, 359(6380), 1146-1151. DOI: 10.1126/science.aap9559.

Wehling, Elisabeth (2016). Politisches Framing. wie eine Nation sich ihr Denken einredet - und daraus Politik macht. Köln, Deutschland: Herbert von Halem Verlag.

Wineburg, Sam; McGrew, Sarah; Breakstone, Joel \& Ortega, Teresa (2016). Evaluating Information. The Cornerstone of Civic Online Reasoning. Abgerufen von https://purl.stanford.edu/fv751yt5934. Abgerufen am 06. August, 2019. 
Wolf, Armin (2013). Wozu brauchen wir noch Journalisten? Wien: Picus.

Wood, Thomas \& Porter, Ethan (2018). The Elusive Backfire Effect. Mass Attitudes' Steadfast Factual Adherence. Political Behavior, 41(1), 135-163. DOI: 10.1007/s11109-018-9443-y.

Zollo, Fabiana; Bessi, Alessandro; Del Vicario, Michela; Scala, Antonio; Caldarelli, Guido; Shekhtman, Louis; Havlin, Shlomo \& Quattrociocchi, Walter (2015). Debunking in a World of Tribes. arXiv: 1510.04267. 
